\title{
A questionnaire survey for psychosocial effects of appearance changes due to cancer treatment and needs for information and supportive care in Japanese cancer patients
}

\section{Hitomi Sakai ${ }^{1}$, Atsuko Koyama², Kaoru Tanaka1, Satomi Watanabe1, Miki Nakura², Toshiko Yasuda², Makiko Hayashi ${ }^{3}$, Miyuki Endo ${ }^{3}$, and Kazuhiko Nakagawa ${ }^{1}$}

1. Department of Medical Oncology, Kindai University Faculty of Medicine

2. Department of Psychosomatic Medicine, Kindai University Faculty of Medicine

3. Department of Nursing, Kindai University Hospital

Patients' characteristics

\section{Background}

Cancer treatment cause appearance change, which leads to psychological, social, and behavioral issues among cancer patients. The Derriford Appearance Scale 59 (DAS59) is a self-report questionnaire designed to assess distress and difficulties experienced by individuals with appearance-related issues. DAS59 has been shown to have excellent reliability and validity, as well as sensitivity to clinical changes ${ }^{1}$. Japanese translated version of DAS59 has been validated ${ }^{2}$.

\section{Patients and method}

We conducted a questionnaire survey using the DAS59 among cancer patients with a prior history of chemotherapy, molecular targeted therapy, or immunotherapy. Patients were recruited from the Departments of Medical Oncology and Psychosomatic Medicine, Kindai University Hospital from June 13, 2018 through September 27, 2018.

\section{DAS59}

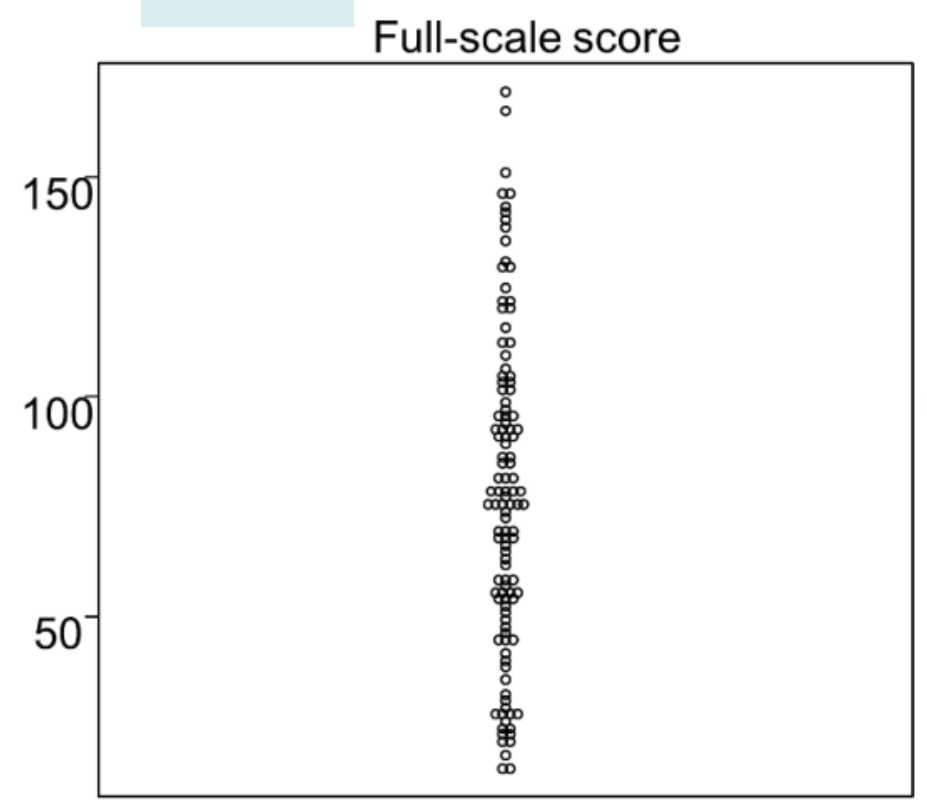

Mean $77.7 \pm 36.4$

Range 16-170
Trial Profile

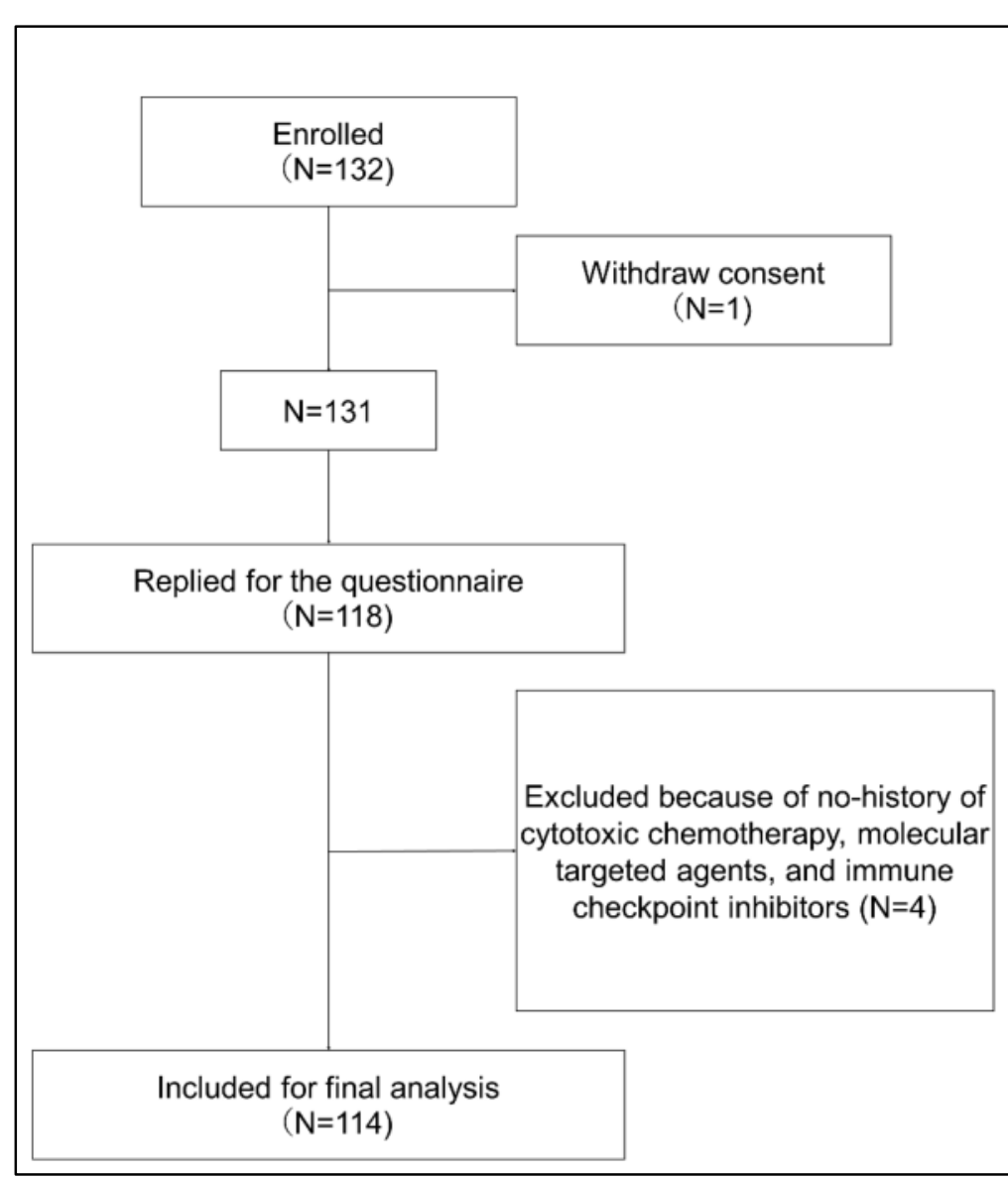

Questionnaire (in Japanese)

DAS59 (score range, 8-262)

Original questions

1. Information needs 2. Support needs

\begin{tabular}{|c|c|c|}
\hline Factor & Group & $\mathrm{N}(\%)$ \\
\hline Age & Mean \pm SD & $62.9 \pm 12.1$ \\
\hline \multirow[t]{2}{*}{ Sex } & Male & $34(29.8)$ \\
\hline & Female & $80(70.2)$ \\
\hline \multirow[t]{3}{*}{ ECOG PS } & 0 & $44(38.6)$ \\
\hline & 1 & $63(55.3)$ \\
\hline & 2 & $7(6.1)$ \\
\hline \multirow{5}{*}{ Cancer } & Breast cancer & 37 (32.5) \\
\hline & Lung cancer & $32(28.0)$ \\
\hline & Colon cancer & $16(14.0)$ \\
\hline & Gastric cancer & $9(7.9)$ \\
\hline & Others & $26(22.8)$ \\
\hline \multirow[t]{2}{*}{ Stage } & Metastatic & $99(86.8)$ \\
\hline & Non-metastatic & $15(13.2)$ \\
\hline \multirow{8}{*}{$\begin{array}{l}\text { Ongoing } \\
\text { treatment }\end{array}$} & Chemo & $39(34.2)$ \\
\hline & Chemo plus molecular & $22(19.3)$ \\
\hline & Molecular & $18(15.8)$ \\
\hline & $\mathrm{ICl}$ & $12(10.5)$ \\
\hline & Chemo plus ICl & $2(1.8)$ \\
\hline & ET plus molecular & $4(3.5)$ \\
\hline & ET & $1(0.9)$ \\
\hline & No treatment & $16(14.0)$ \\
\hline
\end{tabular}

Chemo, chemotherapy; molecular, molecular targeted therapy; $\mathrm{ICI}$, immune checkpoint inhibitor; ET, endocrine therapy
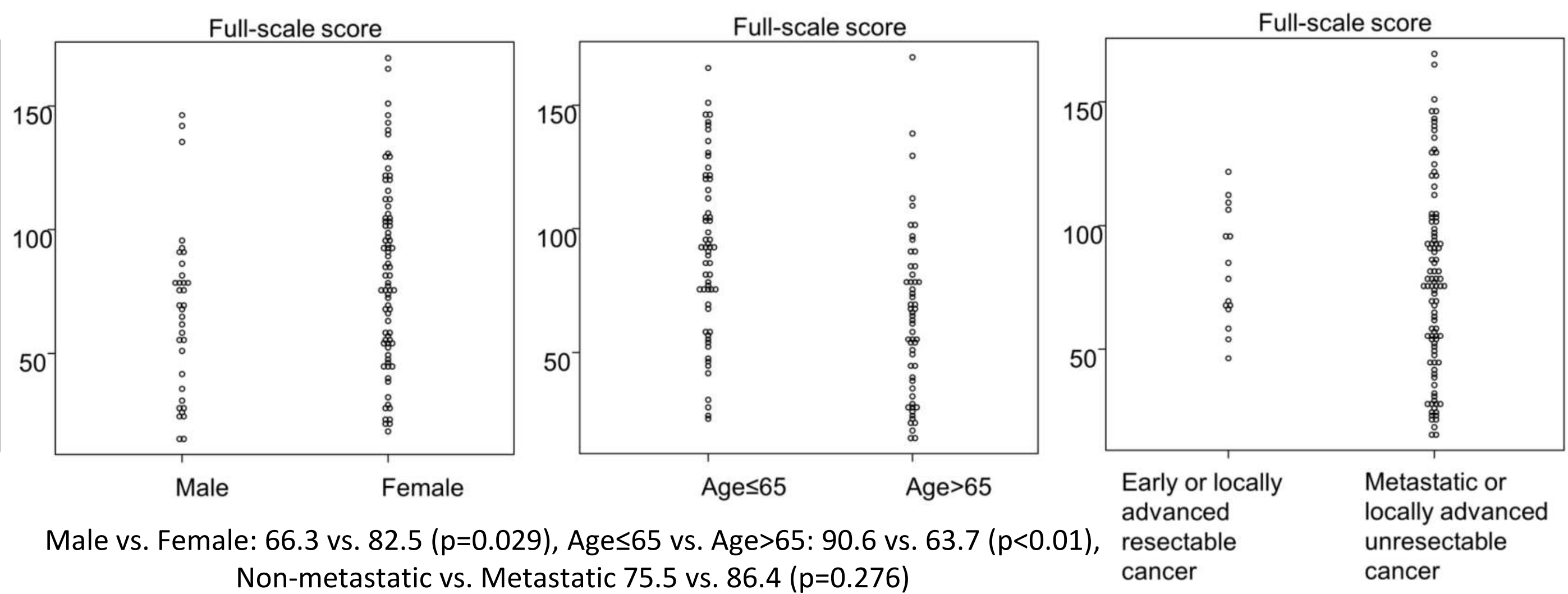

Male vs. Female: 66.3 vs. 82.5 ( $p=0.029)$, Age $\leq 65$ vs. Age $>65: 90.6$ vs. 63.7 ( $p<0.01)$, resectable Non-metastatic vs. Metastatic 75.5 vs. $86.4(p=0.276)$

Information needs at the time of treatment decision-making

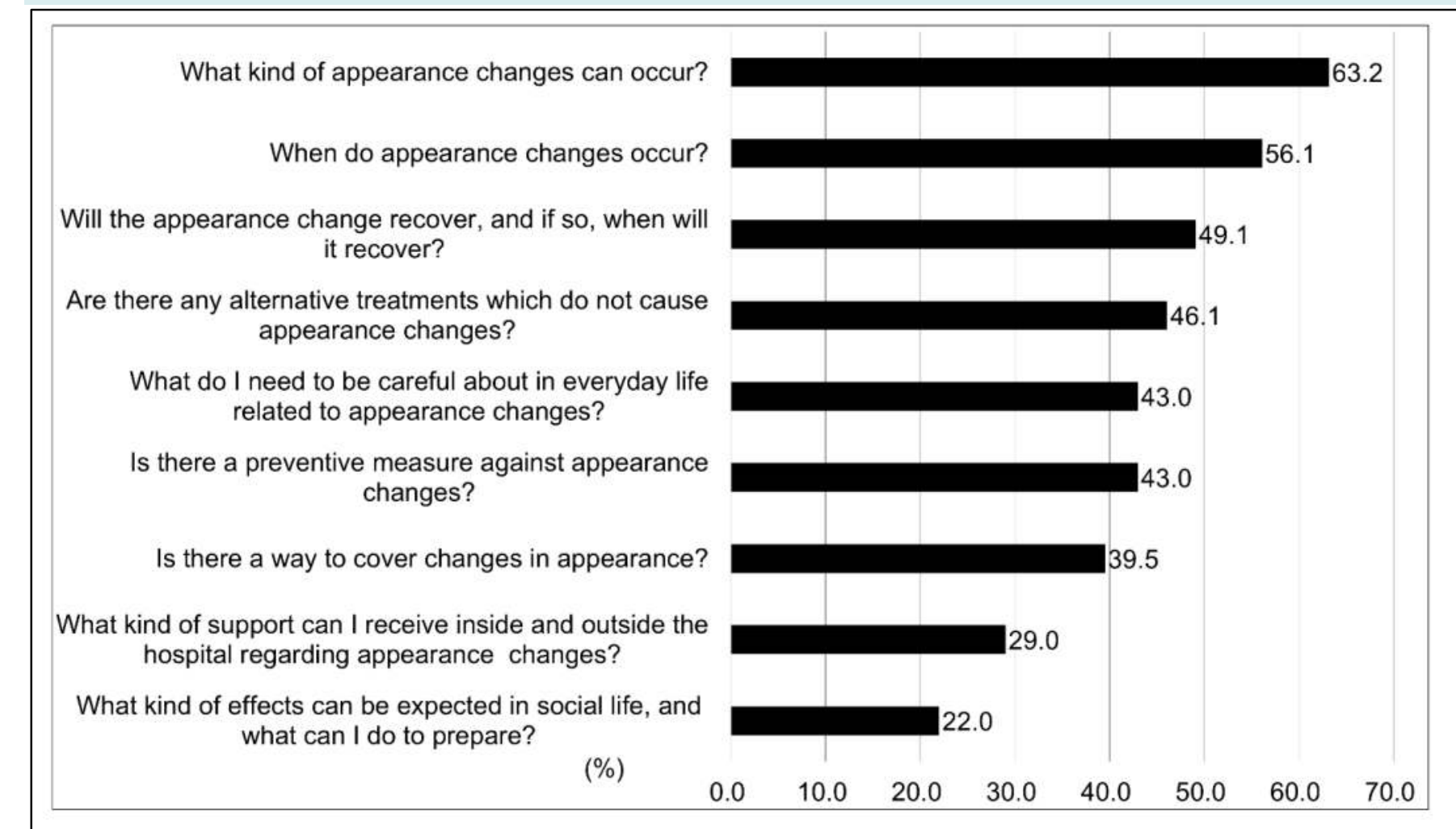

Psychological distress due to appearance changes showed large individual differences. A multidisciplinary approach is required to meet the various needs of cancer patients.
Support needs for appearance changes due to treatment

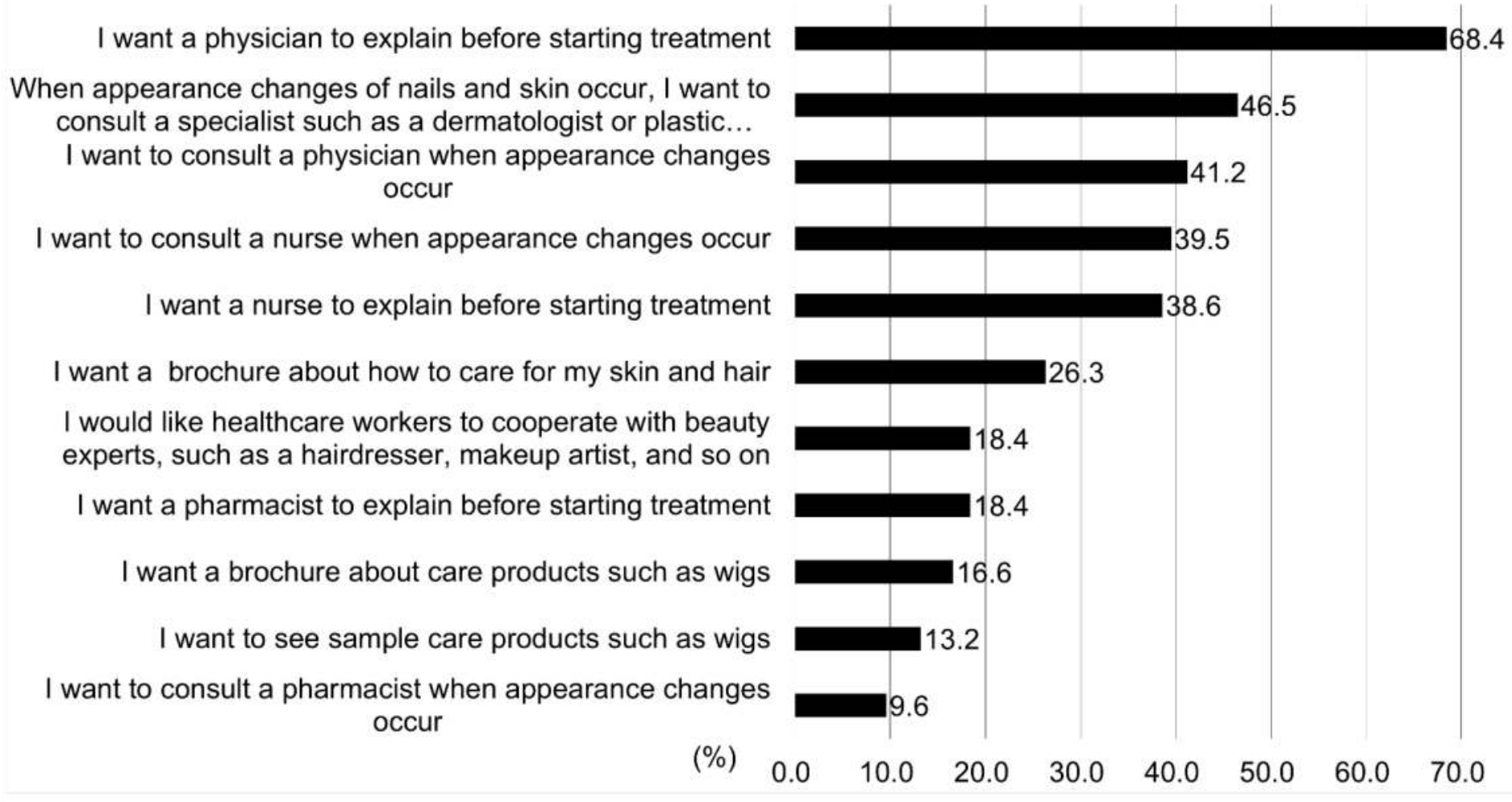

1.Harris DL, Carr AT (2001). Br J Plast Surg 54 (3):216-222 2. Nozawa K. Proceedings of the 72th Annual Convention of the Japanese Psychological Association 2008 (in Japanese) This work was supported by a grant from Kindai University (SR-14). The authors declare no COI. Email: sakai_h@med.kindai.ac.jp 